\title{
Demonstration of Optically Controlled re-Routing in a Photonic Crystal Three-Port Switch
}

Combrié, S.; Heuck, Mikkel; Xavier, S.; Lehoucq, G.; Malaguti, S.; Ballanca, G.; Trillo, S.; Kristensen, Philip Trøst; Mørk, Jesper; de Rossi, A.

Published in:

Advanced Photonics Congress

Publication date:

2012

Document Version

Publisher's PDF, also known as Version of record

Link back to DTU Orbit

Citation (APA):

Combrié, S., Heuck, M., Xavier, S., Lehoucq, G., Malaguti, S., Ballanca, G., Trillo, S., Kristensen, P. T., Mørk, J., \& de Rossi, A. (2012). Demonstration of Optically Controlled re-Routing in a Photonic Crystal Three-Port Switch. In Advanced Photonics Congress (pp. IW3C.5). Optical Society of America.

\section{General rights}

Copyright and moral rights for the publications made accessible in the public portal are retained by the authors and/or other copyright owners and it is a condition of accessing publications that users recognise and abide by the legal requirements associated with these rights.

- Users may download and print one copy of any publication from the public portal for the purpose of private study or research.

- You may not further distribute the material or use it for any profit-making activity or commercial gain

- You may freely distribute the URL identifying the publication in the public portal 


\title{
Demonstration of Optically Controlled re-Routing in a Photonic Crystal Three-Port Switch
}

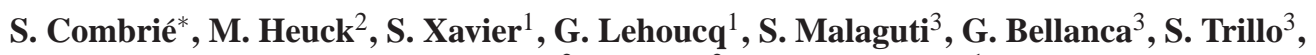 \\ P. T. Kristensen ${ }^{2}$, J. Mørk ${ }^{2}$, and A. de Rossi ${ }^{1}$ \\ ${ }^{1}$ Thales Research and Technology, Palaiseau Cedex FR 91767, France \\ ${ }^{2}$ DTU-Fotonik, Technical University of Denmark, Ørsteds Plads Bygn. 343, 2800 Kgs. Lyngby, Denmark \\ ${ }^{3}$ Department of Engineering, University of Ferrara, Via Saragat 1, IT 44122 Ferrara, Italy \\ *sylvain.combrie@thalesgroup.com
}

\begin{abstract}
We present an experimental demonstration of optically controlled re-routing of a signal in a photonic crystal cavity-waveguide structure with 3 ports. This represents a key functionality of integrated all-optical signal processing circuits.
\end{abstract}

(C) 2012 Optical Society of America

OCIS codes: $130.4815,160.5298$

\section{Introduction}

Photonic crystal $(\mathrm{PhC})$ membrane structures have attracted much attention as a platform for integrated all-optical circuits performing signal processing tasks at ultra high bandwidths with low energy consumption in future optical communication systems [1]. Coupled cavity-waveguide systems provide enhanced non-linear light-matter interaction and small footprint, which are essential for efficient optical integration. Previous work has demonstrated on/off operation in which a control pulse is used to determine the state of the switch $[2,3]$.

In this work, we present a demonstration of a re-routing operation, where a control pulse can switch the signal between two output ports. This constitutes one of the key signal processing functionalities of integrated optical circuits. The left panel in Fig. 1 shows a SEM-image of the 3 port structure that was used in the experiment. It consists of a suspended InP PhC membrane with a bus waveguide and one drop port that couple to an L7 cavity, which is created by removing 7 adjacent holes. The end facets of all ports are provided with tapers to reduce insertion losses [4]. As
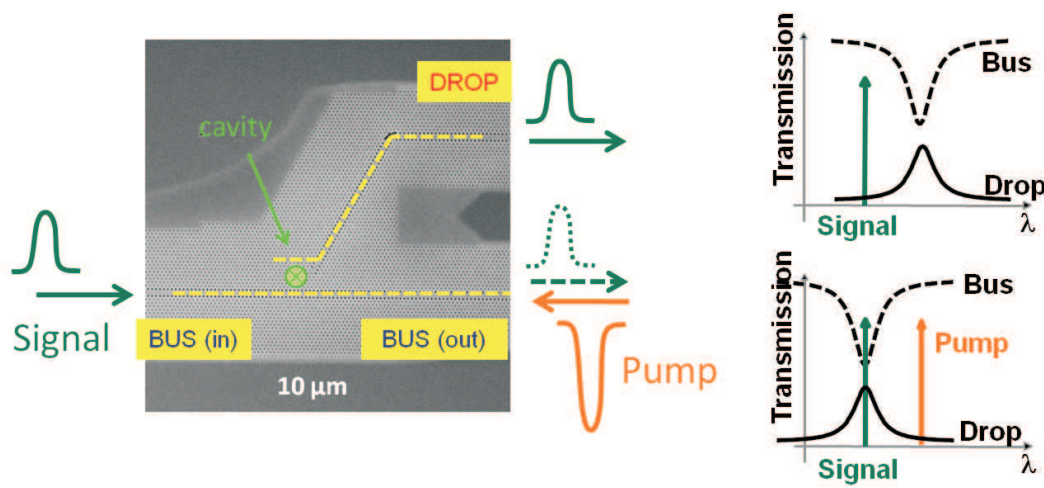

Fig. 1. Left: SEM-image of the PhC structure and a schematic illustration of the input and output fields. Right: Illustration of the switching principle. With the signal red-detuned from the cavity resonance, the transmission through the bus port is large. When the pump is applied, it blue-shifts the cavity resonance through free-carrier dispersion and the transmission through the drop port becomes large while the transmission through the bus is strongly reduced.

illustrated in the upper right panel of Fig. 1, there is a low output from the drop port and a large output from the bus when the signal wavelength is tuned away from the cavity resonance. When the pump is applied, it generates free 
carriers by two-photon absorption, which changes the refractive index by the plasma-effect and thus blue-shifts the cavity resonance. This results in a maximum of the output from the drop and a minimum from the bus, see the lower right panel in Fig. 1. By applying a control pulse, it is thus possible to re-direct the signal from the bus to the drop.

\section{Experiment}

The structure in Fig. 1 is characterized by non-degenerate pump-probe measurements using a setup, which is shown diagrammatically in Fig. 2. The pump and signal pulses originate from the same femto-second mode-locked laser, but pass through tunable filters resulting in different carrier wavelengths $\lambda_{1}$ and $\lambda_{2}$ and pulse widths on the order of $5 \mathrm{ps}$. The signal is modulated at a frequency that is much smaller than the repetition rate of the mode-locked laser to enable lock-in detection. The signal is also passed through a variable delay line to control the difference in arrival times of the signal $t_{\mathrm{S}}$ and pump $t_{\mathrm{p}}$. As sketched in Fig. 1, the pump and signal pulses are injected into opposite ends of the bus

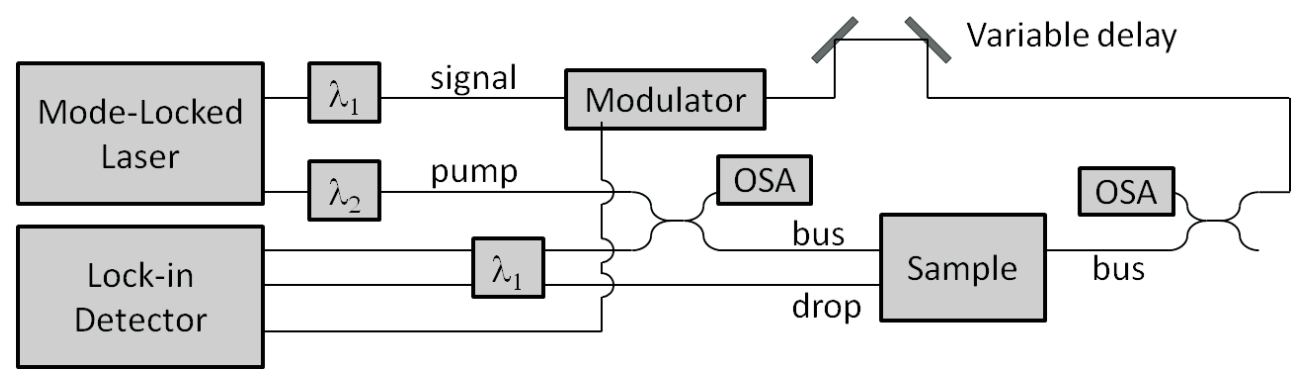

Fig. 2. Diagram of the experimental setup.

to minimize non-linear interactions inside the waveguide, since we are only interested in the effect of the cavity. The signal is collected from either the bus- or the drop output port. Even though the pump is not modulated before entering the sample, the modulation of the signal can be transferred to the pump through the non-linear interaction in the cavity. Therefore, a filter is used before detection to collect only the contribution from the signal.

\section{Results}

The linear transmission properties of the system have been characterized by sending the signal to an optical spectrum analyzer before and after having passed through the sample. The result is shown in the left panel of Fig. 3 (blue
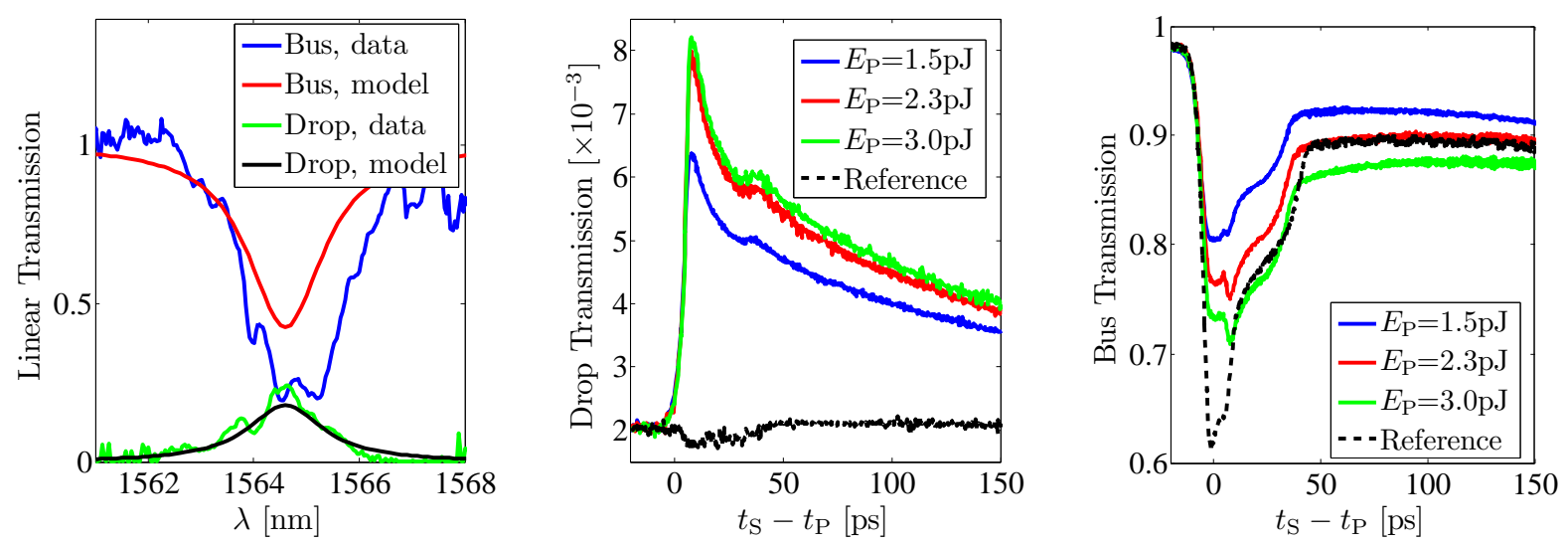

Fig. 3. Left: Linear transmission through the bus (blue) and drop (green) port as well as curves (red and black) calculated from coupled mode theory. Center: Signal transmission through the drop port as a function of the delay between arrival times of the signal $t_{\mathrm{S}}$ and the pump $t_{\mathrm{p}}$ for different pump energies. The black dashed curve is a reference measurement obtained with the pump far red-detuned. Right: Same as the center panel, but for the bus output. 
and green). The figure also shows the calculated transmission from coupled mode theory [5] (red and black) and the agreement is reasonably good when using a loaded quality factor of 1200 . The center (right) panel shows the non-linear transmission of the signal through the drop (bus) port in the presence of a pump pulse as a function of the delay. When the delay has a large negative value, the signal arrives long before the pump, corresponding to linear transmission. These measurements were performed in a wavelength-configuration, where the pump and signal were blue-detuned by $1 \mathrm{~nm}$ and $4 \mathrm{~nm}$ from the cavity resonance, respectively. The black dashed curves are reference measurements with $E_{\mathrm{P}}=$ $3.0 \mathrm{pJ}$ in a configuration where the pump is far red-detuned $(10 \mathrm{~nm})$, in which case its coupling into the cavity should be negligible. The measurements show an increase in the drop transmission and a decrease in the bus transmission when the delay is adjusted so that both the pump and the signal are present in the cavity simultaneously. This clearly demonstrates re-routing of the signal from the bus to the drop. As expected, the effect is dependent on the pump pulse energy $E_{\mathrm{P}}$ and we observe a $6 \mathrm{~dB}$ contrast on the drop transmission and $30 \%$ on the bus at maximum pump energy.

\section{Discussion}

The experiment presented here clearly demonstrates active droppping of an optical signal. The performance could be improved by using a smaller cavity, which would reduce the required pump pulse energy as well as the response time [3]. An optimization of the wavelength-configuration of the signal and pump relative to the cavity resonance is also expected to increase the performance further. Another point that should be addressed in future work is the fact that coupled mode theory predicts a maximum linear drop efficiency of $50 \%$, which can only be improved by more complicated designs e.g. using multiple cavities.

Some details of the bus transmission curves are unexpected, such as the fact that it does not converge to the linear transmission value for large delays. We believe this to be caused by a non-linear interaction taking place in the bus waveguide despite the counter-propagative setup. The reference measurement is meant to probe the non-linear effects of the waveguide, and the fact that it looks similar to the other curves for the bus transmission supports this conclusion. Using a smaller cavity and hence a smaller pump energy would reduce non-linear interactions in the bus waveguide and thereby suppress this unwanted effect.

\section{Acknowledgement}

This work has been supported by the European Union funded project Copernicus, grant no. 249012, as well as the Villum Foundation via the NATEC center.

\section{References}

1. T. Baba, "Remember the Light", Nature Photonics, 1, 11-12 (2007).

2. C. A. Husko, A. de Rossi, S. Combri, Q. V. Tran, F. Raineri, and C. W. Wong, "Ultrafast all-optical modulation in GaAs photonic crystal cavities", Appl. Phys. Lett. 94(2), 021111 (2009).

3. K. Nozaki, T. Tanabe, A. Shinya, S. Matsuo, T. Sato, H. Taniyama, and M. Notomi, "Sub-femtojoule all-optical switching using a photonic-crystal nanocavity", Nat. Photonics 4(7), 477483 (2010).

4. Q. V. Tran, S. Combri, P. Colman, and A. de Rossi, "Photonic crystal membrane waveguides with low insertion losses", Appl. Phys. Lett. 95(6), 061105 (2009).

5. J. D. Joannopoulos and S. G. Johnson and J. N. Winn and R. D. Meade "Photonic Crystals, Molding the Flow of Light", Princeton University Press, (2008). 